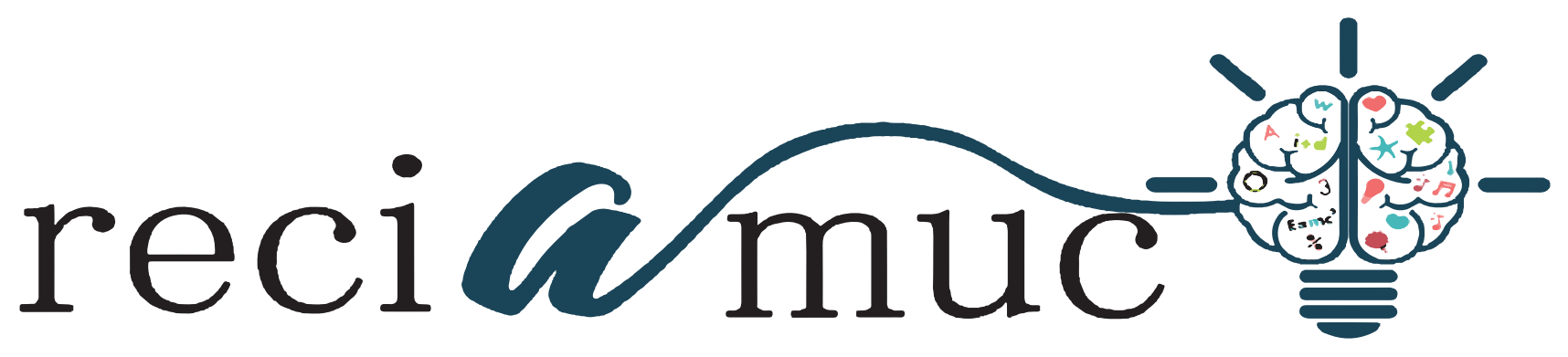

DOI: 10.26820/reciamuc/6.(1).enero.2022.275-283

URL: https://reciamuc.com/index.php/RECIAMUC/article/view/788

EDITORIAL: Saberes del Conocimiento

REVISTA: RECIAMUC

ISSN: 2588-0748

TIPO DE INVESTIGACIÓN: Artículo de revisión

CÓDIGO UNESCO: 5311 Organización y Dirección de Empresas

PAGINAS: $275-283$

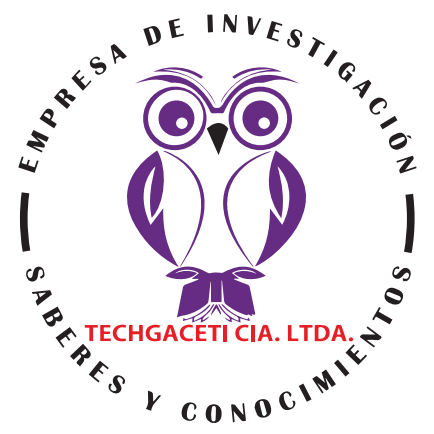

\title{
Las finanzas públicas como herramienta en el desarrollo territorial del cantón Guayaquil
}

Public finances as a tool for the territorial development of the Guayaquil canton

As finanças públicas como ferramenta para o desenvolvimento territorial do cantão de Guayaquil

\section{Pedro Antonio Cedeño Salazar'; Iván Alfredo Morales Maridueña2; Kevin William Guadalupe Sánchez³; Kevin Andrés Sánchez Jiménez ${ }^{4}$}

RECIBIDO: 15/11/2021 ACEPTADO: 05/12/2021 PUBLICADO: 30/01/2022

1. Magister en Finanzas y Economía Empresarial; Economista; Docente de la Universidad de Guayaquil; Guayaquil, Ecuador; eco-pedrocede90@hotmail.com; (iD https://orcid.org/0000-0002-0696-7947

2. Magister en Finanzas; Economista; Docente de la Universidad de Guayaquil; Guayaquil, Ecuador; ivan.moralesm@ug.edu.ec; (ID) https://orcid.org/0000-0002-9527-739X

3. Magister en Economía mención en Desarrollo Económico y Políticas Públicas; Economista; Docente de la Universidad de Guayaquil; Guayaquil, Ecuador; kevin.guadalupe.sanchez@gmail.com; (D) https://orcid.org/00000003-4233-4685

4. Magister en Finanzas; Economista; Docente de la Universidad de Guayaquil; Guayaquil, Ecuador; a_sanchez91@hotmail.com; (D) https://orcid.org/0000-0001-8950-4691

\section{CORRESPONDENCIA}

Pedro Antonio Cedeño Salazar

eco-pedrocede90@hotmail.com

Guayaquil, Ecuador 


\section{RESUMEN}

Las finanzas públicas si son una herramienta de desarrollo en todos los ámbitos, siempre y cuando esta es bien utilizada y hay mecanismos de control para su supervisión. Desarrollo no es solo invertir en servicios públicos, también es el estímulo al fomento de la inversión privada, que entre ambos hacen un binomio perfecto para una mejor calidad de vida y desarrollo territorial, económico, social, entre otros. La metodología utilizada para el presente trabajo de investigación, se enfoca hacia una metodología orientada hacia la necesidad de indagar en forma precisa y coherente una situación. Enmarcada dentro de una revisión bibliográfica de tipo documental, ya que nos vamos a ocupar de temas planteados a nivel teórico como es Las finanzas públicas como herramienta en el desarrollo territorial del cantón Guayaquil. La técnica para la recolección de datos está constituida por materiales impresos, audiovisuales y electrónicos, estos últimos como Google Académico, PubMed, entre otros. La información aquí obtenida será revisada para su posterior análisis. El desarrollo territorial no es solo inversión y mejoramiento de la vialidad, recolección y disposición de desechos sólidos, una mejor distribución o servicio de agua potable, seguridad ciudadana, entre otros, como efectivamente se demuestra en el informe de rendición de cuentas del año 2020 que la municipalidad de Guayaquil invirtió en obras para mejorar la calidad de vida de los ciudadanos y desarrollar más el cantón, aun en la coyuntura de la pandemia por un orden de \$666.646.137,76, habiendo recaudado \$3.694.274.601. Sin embargo, no hay información de nuevos establecimientos de empresas o industrias, ni mucho menos los empleos generados por ellos ni los tributos que estos pagan, sea el sector económico que sea, en este contexto falta ahondar un poco más que y que municipalidad coloque información más actualizada y desagregada, pero lo que sí está claro es que los gobiernos están para proveer calidad de vida y desarrollo.

Palabras clave: Desarrollo, Tributos, Inversión, Gasto, Guayaquil.

\section{ABSTRACT}

Public finances are a development tool in all areas, as long as they are well used and there are control mechanisms for their supervision. Development is not only investing in public services, it is also the encouragement to promote private investment, which between the two makes a perfect combination for a better quality of life and territorial, economic, and social development, among others. The methodology used for this research work focuses on a methodology oriented towards the need to investigate a situation precisely and coherently. Framed within a bibliographic review of documentary type, since we are going to deal with issues raised at a theoretical level such as Public finances as a tool in the territorial development of the Guayaquil canton. The technique for data collection is made up of printed, audiovisual and electronic materials, the latter such as Google Scholar, PubMed, among others. The information obtained here will be reviewed for further analysis. Territorial development is not only investment and improvement of roads, collection and disposal of solid waste, better distribution or service of drinking water, citizen security, among others, as is effectively demonstrated in the 2020 accountability report that the municipality of Guayaquil invested in works to improve the quality of life of citizens and further develop the canton, even in the context of the pandemic for an order of $\$ 666,646,137.76$, having raised $\$ 3,694,274,601$. However, there is no information on new establishments of companies or industries, much less the jobs generated by them or the taxes they pay, whatever the economic sector, in this context it is necessary to delve a little deeper than and that the municipality places information updated and disaggregated, but what is clear is that governments are here to provide quality of life and development.

Keywords: VDevelopment, Taxes, Investment, Expenditure, Guayaquil.

\section{RESUMO}

As finanças públicas são uma ferramenta de desenvolvimento em todas as áreas, desde que sejam bem utilizadas e existam mecanismos de controle para sua fiscalização. Desenvolvimento não é apenas investir em serviços públicos, é também estimular a promoção do investimento privado, que entre os dois faz uma combinação perfeita para uma melhor qualidade de vida e desenvolvimento territorial, econômico, social, entre outros. A metodologia utilizada para este trabalho de investigação centra-se numa metodologia orientada para a necessidade de investigar uma situação de forma precisa e coerente. Enquadrado em uma revisão bibliográfica de tipo documental, pois trataremos de questões levantadas em nível teórico como as finanças públicas como ferramenta no desenvolvimento territorial do cantão de Guayaquil. A técnica de coleta de dados é composta por materiais impressos, audiovisuais e eletrônicos, estes últimos como Google Scholar, PubMed, entre outros. As informações aqui obtidas serão analisadas para posterior análise. O desenvolvimento territorial não é apenas investimento e melhoria de estradas, coleta e disposição de resíduos sólidos, melhor distribuição ou serviço de água potável, segurança cidadã, entre outros, como fica efetivamente demonstrado no relatório de prestação de contas 2020 que o município de Guayaquil investiu em obras para melhorar a qualidade de vida dos cidadãos e desenvolver ainda mais o cantão, mesmo no contexto da pandemia, por uma encomenda de \$666.646.137,76, tendo arrecadado \$3.694.274.601. No entanto, não há informações sobre novos estabelecimentos de empresas ou indústrias, muito menos os empregos gerados por eles ou os impostos que pagam, seja qual for o setor econômico, nesse contexto é necessário se aprofundar um pouco mais e que o município coloque informações atualizado e desagregado, mas o que fica claro é que os governos estão aqui para proporcionar qualidade de vida e desenvolvimento.

Palavras-chave: Desenvolvimento, Impostos, Investimentos, Despesas, Guayaquil. 


\section{Introducción}

El Desarrollo Económico Local (DEL), es un enfoque que ha surgido acorde a los problemas coyunturales económicos y sociales que se han presentado recientemente en los diferentes países; pues, estas dificultades se han incrementado de acuerdo a diversos factores, tales como: Cambios en el mercado laboral, el incremento de la economía informal, la inexistencia de ventajas competitivas, y la falta de políticas descentralizadas por parte de los gobiernos de turno; estas características han hecho como consecuencia la necesidad de fomentar un nuevo tipo de desarrollo económico enfocado en el crecimiento endógeno, en la generación de competitividad, un cambio estructural, en lo que respecta al ámbito territorial y en el bienestar de una sociedad local (Chacha, Alvarado, Villacís, \& Flores, 2019, pág. 85).

Se puede señalar que las finanzas públicas son un conjunto de medidas y políticas adoptadas por el Estado que determinan el sistema financiero público, de gran influencia en el sistema económico pues lo dinamizan y regula, y que difieren de las finanzas privadas por sus fines orientados al bien común. Así la actividad tributaria es fundamental pues permite la obtención de recursos que serán redistribuidos en obras desde la centralidad o la localidad. Por lo expuesto, es preciso recalcar que en el manejo de las finanzas públicas se cuenta con un instrumento fundamental que armoniza los ingresos, gastos e inversión denominado presupuesto (Jaramillo Yaguachi, 2014, pág. 15).

El desarrollo local se presenta como un generador de proceso de edificación social a nivel local, que muestra como finalidad agrupar los recursos del territorio atreves de proyectos en común e incluir al conjunto de la población, incorporando los diferentes rasgos sociales, políticos, ambiental, cultural que requiere de la participación del Gobierno Autónomo Descentralizado, ente encargado de cumplir el rol suministrado por el gobierno nacional, teniendo como responsabilidad de generar bienestar común a la sociedad por las demandas que estos presenten (Peralta Albán, 2021, pág. 3).

\section{Principales teorías de desarrollo econó- mico y social}

- Teoría de la modernización. La teoría de la modernización establece que las sociedades modernas son más productivas, los niños están mejor educados y los necesitados reciben más beneficios.

- Teoría de la dependencia. Las bases de la teoría de la dependencia se originaron en 1950 como resultado de las investigaciones de la Comisión Económica para América Latina y el Caribe (CEPAL). La teoría de la dependencia está compuesta por 4 puntos fundamentales: a) desarrollar una considerable demanda interna efectiva en términos de mercados nacionales; b) reconocer que el sector industrial es sustancial para alcanzar mejores niveles de desarrollo nacional, especialmente porque este sector crea valor agregado a los productos en comparación con el sector agrícola; c) incrementar los ingresos de los trabajadores como medio para generar mayor demanda agregada dentro de las condiciones del mercado nacional; d) promover un papel gubernamental más efectivo para fortificar las condiciones de desarrollo nacional y aumentar los estándares de vida del país.

- Teoría de los sistemas mundiales. Los principales supuestos de la teoría de los sistemas mundiales establecen que: a) hay un fuerte vínculo entre las ciencias sociales, especialmente entre la sociología y las disciplinas económicas y políticas. Esta escuela reconoce que generalmente se le da una mayor atención al desarrollo individual de cada una de estas disciplinas que a la interacción entre ellas, y cómo éstas interacciones afectan en términos reales las condiciones nacionales de una sociedad; b) en

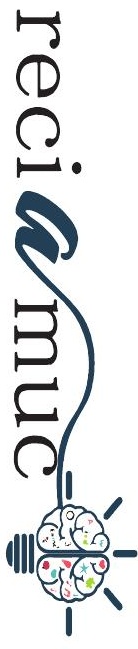


vez de dirigir el análisis a cada una de las variables, es necesario estudiar la realidad de los sistemas sociales; y c) es necesario reconocer el nuevo carácter del sistema capitalista.

- Teoría de la globalización. Los principales supuestos que se extraen de la teoría de la globalización se resumen en tres puntos fundamentales. Primero, los factores culturales son los aspectos determinantes de las sociedades. Segundo, bajo las condiciones mundiales actuales no es importante utilizar a las naciones-estados como unidad de análisis, ya que la comunicación global y los vínculos internacionales están haciendo a esta categoría menos útil. A medida que haya una mayor estandarización de los avances tecnológicos una mayor cantidad de sectores sociales podrán conectarse con otros grupos alrededor del mundo. Esta situación incluye a los grupos dominantes y no dominantes de cada país (Aragón Lozano, 2019, págs. 5-7).

El Gobierno Autónomo Descentralizado del cantón de Guayaquil se encuentra ubicado en la provincia del Guayas en la zona 8 de planificación de la Secretaría Nacional de Planificación y Desarrollo (SENPLADES), esta entidad pública realiza diferentes inversiones en proyectos como infraestructura, regeneración pública, salud, educación, entre otros con el fin de amplificar su capacidad para la prestación de servicios y producción de bienes, proporcionando así el bienestar de sus habitantes e impulsar el desarrollo atreves de ordenanzas y resoluciones (Peralta Albán, 2021, pág. 2).

Para promover el desarrollo integro en cada cantón, basado en la ejecución de tareas y obras además de la prestación de servicios públicos es necesario disponer de recursos económicos en gran cuantía; como tal cada Gobierno Autónomo Descentralizado de los cantones que componen las provincias de nuestro país están llamados continuamente en fortalecer su capacidad fiscal. La recaudación tributaria en el GAD Municipal del cantón Guayaquil se realiza a los sujetos pasivos, es decir a aquellas personas que deben pagar los diferentes impuestos municipales como una contribución, debido a que son propietarios de bienes inmuebles como terrenos, vivienda, oficina, edificio o aquellos que ejercen actividades de carácter económico; considerando que también se realiza la recaudación de otros impuestos municipales y metropolitanos como el cobro de tasas y contribuciones especiales de mejoras para cumplir con las disposiciones que establece la ley (Vera De la Cruz, 2021, págs. 3-4).

\section{Metodología}

La metodología utilizada para el presente trabajo de investigación, se enfoca hacia una metodología orientada hacia la necesidad de indagar en forma precisa y coherente una situación. Enmarcada dentro de una revisión bibliográfica de tipo documental, ya que nos vamos a ocupar de temas planteados a nivel teórico como es Las finanzas públicas como herramienta en el desarrollo territorial del cantón Guayaquil. La técnica para la recolección de datos está constituida por materiales impresos, audiovisuales y electrónicos, estos últimos como Google Académico, PubMed, entre otros. La información aquí obtenida será revisada para su posterior análisis. 


\section{Resultados}

\begin{tabular}{lrrr}
\hline Programas sociales & 2019 & 2020 & \multicolumn{1}{c}{$\begin{array}{c}\text { Variación } \\
\text { en porcentaje }\end{array}$} \\
\hline Campaña de fumigación y desratización & 1357321,76 & 1357321,76 & 0,00 \\
Plan ambiental & 1328673,79 & 1639786,95 & 23,42 \\
Plan más alimento- red de mercados & 966689,41 & 6681708,37 & 591,19 \\
Cultura y promoción civica y deportes & 18127292,16 & 7060738,36 & $-61,05$ \\
Proyectos de áreas verdes y parques & 10040539,98 & $1074,0539,38$ & 6,97 \\
Plan más seguridad & 7410000,00 & 11988837,06 & 61,79 \\
Plan más salud e higiene & $14,186953,22$ & 13539345,11 & $-4,56$ \\
Transporte y turismo & 4989086,88 & 15373551,69 & 208,14 \\
Servicio a la comunidad seguridad y limpieza & $14,916463,29$ & 20759515,12 & 39,17 \\
Plan de acción social y educación & 41450850,95 & 35153001,53 & $-15,19$ \\
Total asignado al presupuesto social & $114,773871,44$ & $124,294345,33$ & 8,29 \\
Incremento del gasto social del municipio & & 9520473,89 & \\
\hline
\end{tabular}

Imagen 1. Guayaquil: presupuesto municipal asignado en 2019 y 2020

Fuente: (Chávez Páez, 2021)

Como se puede ver, el presupuesto asignado para temas de protección social debido al COVID-19 se incrementó en: plan ambiental $(23,42 \%)$, servicio a la comunidad seguridad y limpieza $(39,17 \%)$, y plan más seguridad $(61,79 \%)$ para cuidar la ciudad y vigilar de cerca el riesgo de contagio. Sin embargo, los mayores incrementos de dos cifras del presupuesto 2020 comparado con 2020, correspondieron a: plan más alimento-red de mercados en un 591,19\% (en el que se cuenta la entrega de kits alimenticios a familias vulnerables que no podían salir a trabajar para obtener sus ingresos en el día a día, tanto en la parte urbana como rural del cantón), y en transporte y turismo en un $208,14 \%$. El gasto social de la Municipalidad de Guayaquil se incrementó en $8,29 \%$ lo cual equivale a \$9 520473 que fueron reasignados a lo social como gasto prioritario entre los gastos municipales. En conclusión, el gasto de protección social llegó a ser \$124 294345 de un presupuesto total de \$834 800999 que representa el 14,89\% (Chávez Páez, 2021, pág. 48).

\begin{tabular}{|lcccc|}
\hline Ingresos & $\begin{array}{c}\text { Presupuesto } \\
\text { inicial 2019 }\end{array}$ & $\begin{array}{c}\text { Peso \% de cada } \\
\text { fuente de ingresos }\end{array}$ & $\begin{array}{c}\text { Presupuesto } \\
\text { inicial 2020 }\end{array}$ & $\begin{array}{c}\text { Peso } \% \text { de cada } \\
\text { fuente de ingresos }\end{array}$ \\
\hline Ingresos propios & 185807300,00 & $23 \%$ & 180775330,46 & $22 \%$ \\
Asignaciones GAD's & 297553700,00 & $36 \%$ & 297500000,00 & $36 \%$ \\
Ingresos de financiamiento & 333600000,00 & $41 \%$ & 356525669,54 & $43 \%$ \\
Total & 816961000,00 & $100 \%$ & 834801000,00 & $100 \%$ \\
\hline
\end{tabular}

Imagen 2. Guayaquil: fuentes de financiamiento del presupuesto, 2019-2020

Fuente: (Chávez Páez, 2021)

El Municipio de Guayaquil cubre sus gastos con los ingresos que recibe, de los cuales el $22 \%$ provienen de ingresos propios (tributos municipales), 36\% de asignaciones del gobierno central al municipio, y el mayor componente son los "ingresos de financiamiento" en los que se concentran un $43 \%$ de los ingresos. Ninguno de estos 3 rubros sobrepasa el $50 \%$ por lo que puede decirse que la Municipalidad no depende en exce- 
so ni de las asignaciones recibidas del gobierno central ni de los ingresos de financiamiento (Chávez Páez, 2021, pág. 58).

\begin{tabular}{|c|c|}
\hline Ingresos inelásticos & Valores codificados a junio 2020 \\
\hline A los predios urbanos & $\$ 13000000,00$ \\
\hline A los predios rústicos & $\mathbf{\$ 1} 000000,00$ \\
\hline Recolección de basura & $\$ 19000000,00$ \\
\hline Apertura, pavimentación, ensanche y construcción & $\$ 12700000,00$ \\
\hline Del sector privado no financiero & 5985000,00 \\
\hline Infracción a ordenanzas municipales & $\$ 280000,00$ \\
\hline Otras concesiones & $\$ 1630000,00$ \\
\hline Otros no especificados & $\$ 4,000930,46$ \\
\hline Bonos del Estado & $\mathbf{\$ 1 5} 000000,00$ \\
\hline Del fondo de descentralización a municipios & $\mathbf{s} 80770195,52$ \\
\hline Fondos de autogestión & $\$ 98,499506,95$ \\
\hline Otros títulos & $\$ 10380000,00$ \\
\hline De anticipo por devengar de ejercer de GADS y empresas & $\$ 20864,273,61$ \\
\hline Anticipos por devengar & $\$ 10755000,00$ \\
\hline Cuentas por cobrar & $\$ 125010,00$ \\
\hline Ingresos municipales más inelásticos ante COVID-19 & $\$ 288989916,54$ \\
\hline Total ingresos municipales & 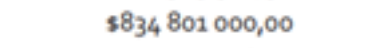 \\
\hline \% de ingresos inelásticos & $34,62 \%$ \\
\hline
\end{tabular}

Imagen 3. Guayaquil: porcentaje de ingresos inelásticos, junio de 2020

Fuente: (Chávez Páez, 2021)

- El 2019 con el 2020 se aprecia una caída de aproximadamente $20 \%$ en la recaudación de los tributos municipales en diversos rubros, tanto los que están ligados a la propiedad como los que están ligados a la actividad económica. Sin embargo, los más elásticos son los que están ligados a la actividad económica, en los cuales se nota una merma de $20 \%-25 \%$ si comparamos 2020 con 2019.

- Otros tributos cuya recaudación ha disminuido, son los relacionados con transferencias de dominios, es decir donde hay una compra venta, aportes de bienes a sociedades, acciones de pago, cualquier transferencia de dominio paga el impuesto de alcabalas y también hay un impuesto de transferencia de dominio sobre la compra venta de bienes urbanos que graba en un $10 \%$ a la utilidad entre el precio de compra y el precio de venta.
- Otro tributo que ha experimentado una fuerte caída obviamente es el de espectáculos públicos. Los espectáculos están funcionando muy limitadamente, la normativa actual es de $30 \%$ de aforo y la caída de este impuesto es alrededor de $75 \%$, no se llega en 2020 al $\$ 1.000 .000$ cuando generalmente se recaudaba alrededor de $\$ 4.000 .000$.

- Los impuestos más inelásticos han sido los impuestos prediales. En Guayaquil se recaudan alrededor 28000000 de dólares y al finalizar 2020 se han recaudado 25000000 y es difícil incrementar esa recaudación ahora porque realmente el grueso del impuesto se lo cobra en enero y febrero -porque hay un incentivo de descuento los demás meses el cobro es menor-. En la recaudación de este impuesto hay una caída de aproximadamente 2500 000, y en ese impuesto está incluido el cobro de las Contribuciones Especial de Mejoras (CEM) (Chávez Páez, 2021, págs. 59-60). 


\section{Informe de rendición de cuentas 2020-2021}

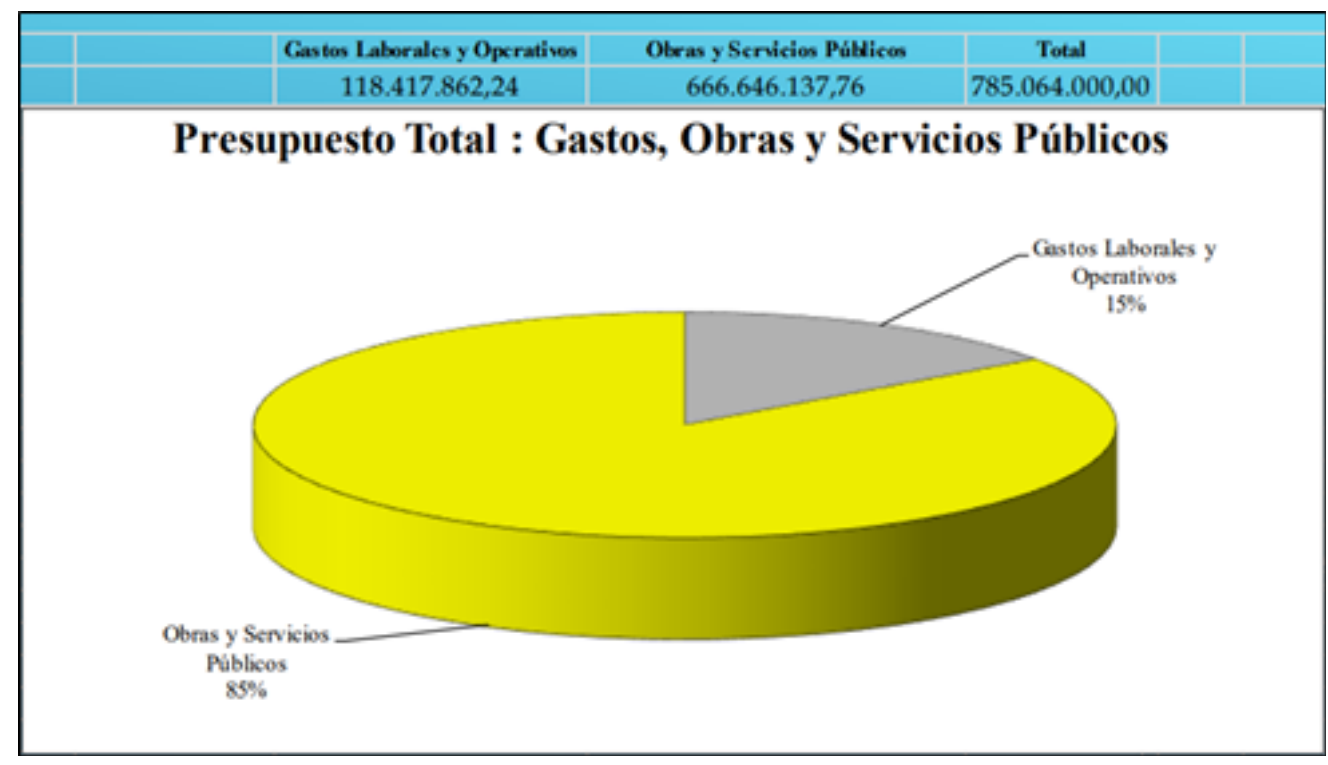

Imagen 4. Gastos obras y servicios públicos.

Fuente: (GAD Municipal Guayaquil, 2021)

\begin{tabular}{|c|c|c|}
\hline Tipo de servicio & Beneficiarios directos & Empleos generados \\
\hline \multicolumn{3}{|c|}{ Vialidad } \\
\hline $\begin{array}{l}\text { - } \text { Nuevas vías } 176,60 \mathrm{Km} \\
\text { - } 227 \mathrm{Km} \mathrm{de} \mathrm{vías} \\
\text { mantenidas. }\end{array}$ & 1.09 millones de habitantes. & 25.530 \\
\hline \multicolumn{3}{|c|}{ Infraestructura de servicios } \\
\hline $\begin{array}{l}\text { - Mant. y sum. de generadores } \\
\text { - Instalación sistemas de } \\
\text { iluminación }\end{array}$ & $\begin{array}{c}\text { 4.501.656 millones de } \\
\text { habitantes }\end{array}$ & 500 \\
\hline \multicolumn{3}{|c|}{ Riesgo } \\
\hline $\begin{array}{l}\text { - Bóvedas y túmulos de } \\
\text { cementerios } \\
\text { - Escalinatas }\end{array}$ & 370.000 habitantes & 2.883 \\
\hline \multicolumn{3}{|c|}{ Transporte } \\
\hline $\begin{array}{l}\text { - Const. de paradas de } \\
\text { metrovía } \\
\text { - Mantenimiento vial }\end{array}$ & 437.624 habitantes & 900 \\
\hline \multicolumn{3}{|c|}{ Urbe habitable } \\
\hline $\begin{array}{l}\text { - Reconformación } \\
\text { - Infraestructural vial }\end{array}$ & 5.565 habitantes & 300 \\
\hline
\end{tabular}

Fuente: (GAD Municipal Guayaquil, 2021) 
- 69.951.069,13 \$ cancelados por concepto de servicio de recolección de desechos sólidos no peligrosos de la ciudad y sus parroquias rurales, limpieza del estero salado y su disposición final en el relleno sanitario "Las Iguanas". Donde se recolectaron 1.512.134,23 toneladas entre desechos sólidos y limpieza y mantenimiento de esteros salados, de las cuales fueran dispuestas en el relleno sanitario 1.55.058,85 toneladas, beneficiando aproximadamente a 3.000.000 millones de habitantes con una cobertura del $98 \%$.

- Se recaudaron 2.976.645,50 \$ por mercados mayoristas, minoristas, bahías y áreas de ordenamiento. Así como en donde se asignaron un total de 720 nuevos puestos en mercados, bahías y terminal de trasferencia de víveres.

- Se empezó la construcción de un nuevo mercado mayorista, que tiene prevista su inauguración para el año 2021 con una inversión de \$1.319.555,89. Así como un nuevo mercado minorista de mariscos con una inversión de \$1.765.471., (GAD Municipal Guayaquil, 2021).

Datos actualizados por el Servicios de Rentas Internas (SRI) (2022) el apartado de Recaudación Nacional por domicilio fiscal e impuesto hasta el año 2021, el cantón de Guayaquil había recaudado por concepto de tributos internos o impuestos municipales $\$ 4.022 .835 .387$ con un aumento del 8,89\% con respecto al año 2020 cuando recaudaron \$3.694.274.601.

\section{Conclusiones}

Se estuvo revisando el portal de la Municipalidad de Guayaquil, sin embargo, no hay un informe de rendición de cuentas más resumido como el del año 2020, solo se destaca un video en donde hablan de la gestión 2020/2021. En el Sistema de recaudación interna, solo existe información de la totalidad de impuestos recaudados hasta el año 2021 por cantón y provincia, sim embargo cuando se busca la información más detallada por actividad económica, esta no está por cantón ni provincia, sino a manera general. Estas aseveraciones se hacen ya que las finanzas públicas municipales o cantonales vienen por dos vías, una, los recursos que envía el gobierno nacional ecuatoriano y otro por concepto de recaudación de tributos o impuestos municipales.

El desarrollo territorial no es solo inversión y mejoramiento de la vialidad, recolección y disposición de desechos sólidos, una mejor distribución o servicio de agua potable, seguridad ciudadana, entre otros, como efectivamente se demuestra en el informe de rendición de cuentas del año 2020 que la municipalidad de Guayaquil invirtió en obras para mejorar la calidad de vida de los ciudadanos y desarrollar más el cantón, aun en la coyuntura de la pandemia por un orden de $\$ 666.646 .137,76$, habiendo recaudado $\$ 3.694 .274 .601$. Sin embargo, no hay información de nuevos establecimientos de empresas o industrias, ni mucho menos los empleos generados por ellos ni los tributos que estos pagan, sea el sector económico que sea, en este contexto falta ahondar un poco más que y que municipalidad coloque información más actualizada y desagregada, pero lo que sí está claro es que los gobiernos están para proveer calidad de vida y desarrollo.

\section{Bibliografía}

Aragón Lozano, E. J. (2019). El desarrollo socioeconómico de la parroquia rural El Morro, del cantón Guayaquil 2010-2018.

Chacha, S. A., Alvarado, E., Villacís, C., \& Flores, O. (2019). Desarrollo económico local en Ecuador: Relación entre producto interno bruto y sectores económicos. Revista de ciencias sociales, 25(1), 82-98.

Chávez Páez, W. (2021). Informe de diagnóstico de recuperación económica urbana y resiliencia en Guayaquil. CEPAL.

GAD Municipal Guayaquil. (2021). Rendiciòn de cuentas 2020. 
Jaramillo Yaguachi, J. D. (2014). Presupuesto participativo: aplicación en el marco de las finanzas públicas y la participación ciudadana.

Peralta Albán, J. M. (2021). Análisis del desarrollo local generado por el Gobierno Autónomo Descentralizado del cantón Guayaquil. Período 20102018 .
SRI. (03 de 02 de 2022). Obtenido de https://www.sri. gob.ec/estadisticas-generales-de-recaudacion-sri

Vera De la Cruz, K. P. (2021). Análisis de la cultura tributaria en relación a la recaudación del impuesto predial urbano en el cantón Guayaquil 2021.

\section{CITAR ESTE ARTICULO:}

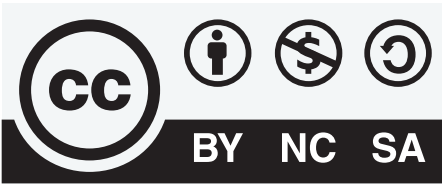

Cedeño Salazar, P. A., Morales Maridueña, I. A., Guadalupe Sánchez, K. W., \& Sánchez Jiménez, K. A. (2022). Las finanzas públicas como herramienta en el desarrollo territorial del cantón Guayaquil. RECIAMUC, 6(1), 275-283. https:// doi.org/10.26820/reciamuc/6.(1).enero.2022.275-283 